\title{
A STUDY ON WATER HYDRAULIC FIRE-FIGHTING ROBOT
}

\author{
Liang ZHANG, Ato KITAGAWA, Hideyuki TSUKAGOSHI \\ Department of Control and Systems Engineering, Tokyo Institute of Technology \\ 2-12-1 Ookayama, Meguro-ku, Tokyo, 152-8552 Japan \\ (E-mail: lzhang@ctrl.titech.ac.jp)
}

\begin{abstract}
Fire-fighting has long been a dangerous task to man, so robots have been developed to fight fire. Up to now, the fire-fighting robots have mainly been driven by electric power and have reliability problems when exposed to fire. So, we propose to make a water hydraulic fire-fighting robot. The proposed robot, which mainly consists of 10 leg-wheels, can run along flat ground, climb steps, go directly into firing places. The wheels can run fast along flat places and the legs can go up and down. The wheels are driven by two water hydraulic motors and the legs are driven by water hydraulic cylinders. The mechanism and the control method of the robot are discussed in this paper.
\end{abstract}

\section{KEY WORDS}

Water hydraulics, Fire-fighting robot, Leg-wheel robot

\section{Introduction}

Fire fighting activity in the fire spot has long been a tedious and dangerous task for fireman. Therefore it becomes a dream of long time of man to develop remote-control fire-fighting robots. To cope with the complicated fire spots, a fire-fighting robot must have heat-resisting, waterproofing, ease-turning and obstaclecrossing functions.

Up to now, the fire-fighting robots, which are mainly driven by electric power ${ }^{[1]}$, have reliability problems when exposed to fire and can not meet all the above demands. So, we propose to build a water hydraulic fire-fighting robot.

The "water hydraulic" here means that the pressure medium of a fluid power system is water instead of oil in conventional hydraulic systems. The history of water hydraulics was comparatively old, but the leading medium of fluid power systems was turned into oil from water because of the weak points of water hydraulics such as rustiness, lubrication, leakage, etc. In recent years with the development of science and technology, the defects of water hydraulics have gradually been overcome and products for tap water use have been coming into market ${ }^{[2]}$. With the rising concern for environmental problems, water, due to its 
pollution-free, fire-resistance, low cost, has again been paid attention to. On this account, a water hydraulic fire-fighting robot was considered.

The proposed robot, which mainly consists of 10 leg-wheels, can run along flat ground, climb steps, go directly into firing places. The wheels can run fast along flat places and the legs can go up and down. The wheels are driven by two water hydraulic motors that are controlled through 2 water hydraulic servo valves. The legs are driven by water hydraulic cylinders. The appearance of the robot is shown in Figure 1 and there is also an outer cover for the robot as shown in dotted lines. The mechanism and the control method of the robot are discussed in this paper.

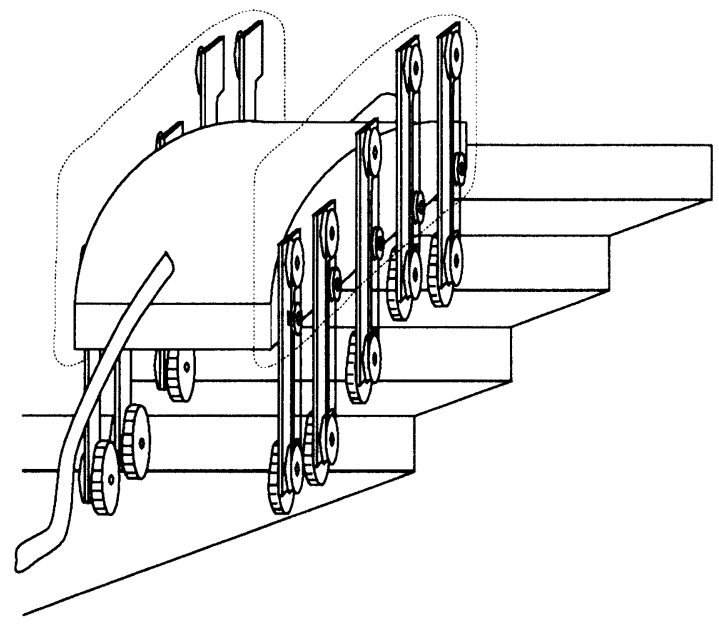

Figure 1 Appearance of the robot

\section{Request performance and design strat- egy of the robot}

- A heat-resistant function is necessary in order to move through firing places.

Because ignitable oil or electricity is not suitable as power source, high pressure water (7 $\mathrm{MPa}$ ) is used.

- An easy turning and fast running ability is necessary in order to cope with emergency fire.

A wheel type is more effective compared with a crawler type ${ }^{[3]}$.

- Obstacle crossing ability is necessary in order to be adaptive to various kinds of fire spots.

Fire spots may not be only flat topography, and may have various kinds of obstacles which the robot has to get over to reach the fire spots.
So the robot is built with 10 leg-wheels, each of which is equipped with a water hydraulic cylinder. A leg wheel will be lifted up by the cylinder to get over an obstacle that is higher than the diameter of a wheel. The head sides of ten cylinders are connected to form communicating tubes.

- The use of electricity needs to be refrained from in order to increase the reliability of the robot.

The sensors that are driven by electricity should be used as few as possible.

- Cost should be cut down.

At the present time the price of water hydraulic products is very high. In order to reduce the cost of the whole system, only two water hydraulic motors are used to drive the robot.

\section{Mechanism consideration}

\subsection{Outline of the robot}

A figure of outline of the robot is shown in Figure 2. The robot is mainly composed of $10 \mathrm{leg}$-wheels ( 5 for each side) that can get over obstacles by moving legs up and down, and a water gun for fire extinguishing is also equipped at the front of the robot.

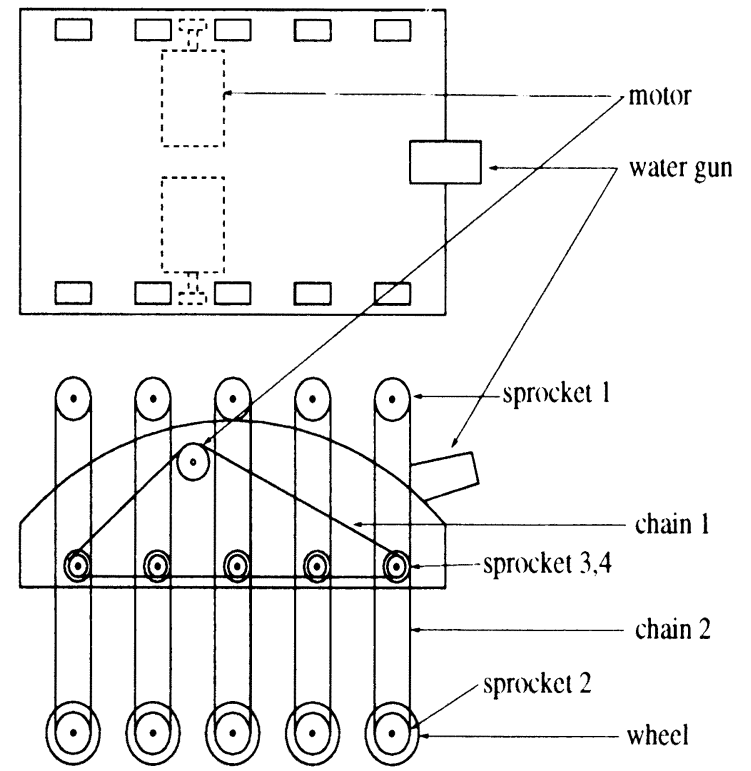

Figure 2 Outline of the robot

\subsection{Drive system}

Two water hydraulic motors are used to drive 10 wheels, one for 5 wheels of one side by chains and 
sprockets. A wheel will be lifted up in order to get over an obstacle that is higher than the diameter of a wheel. Therefore a motor and wheels can not be directly connected by only one chain. So as shown in Figure 2, sprocket 1 and 2, which can move with a leg, are installed to the top and the bottom of each leg. First, the rotation of motor is conveyed to sprocket 3 and 4 by chain 1 , and then to wheels by chain 2, sprocket 1 and 2 .

\subsection{Communicating tubes}

In order for the whole robot to move smoothly, the head sides of 10 water hydraulic cylinders, which are filled with water, are connected to act as communicating tubes as shown in Figure 3.

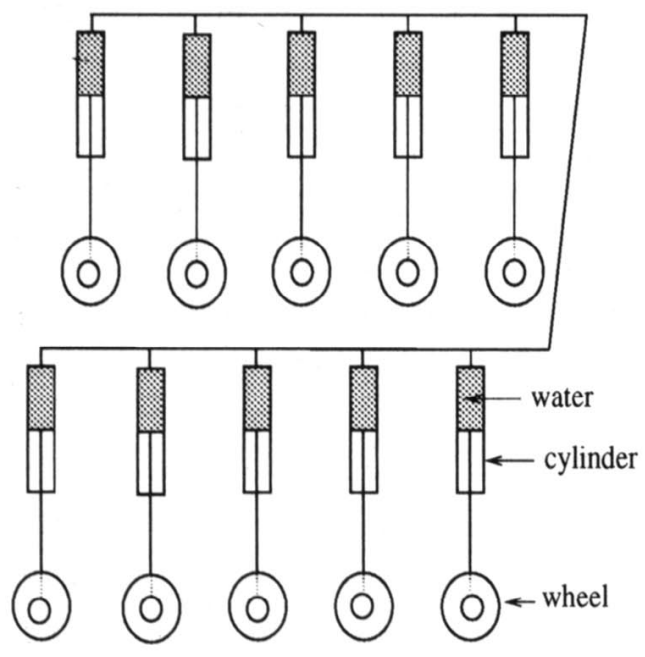

Figure 3 Communicating tubes

\subsection{Leg mechanism}

\subsubsection{Structure of a leg}

Structure of a leg is shown in Figure 4. When a bumper collides with an obstacle, a leg (the part that is not colored in the figure) can be lifted up along the body (the part that is colored in the figure) through a water hydraulic cylinder, the rod side of which is connected to the movable leg.

\subsubsection{Installation method of a cylinder}

A cylinder is installed to the main body in such a way as shown in Figure 5 so that the rod of cylinder may not directly receive the force that comes from the collision between a wheel and an obstacle. Therefore trunnion cylinders are used.

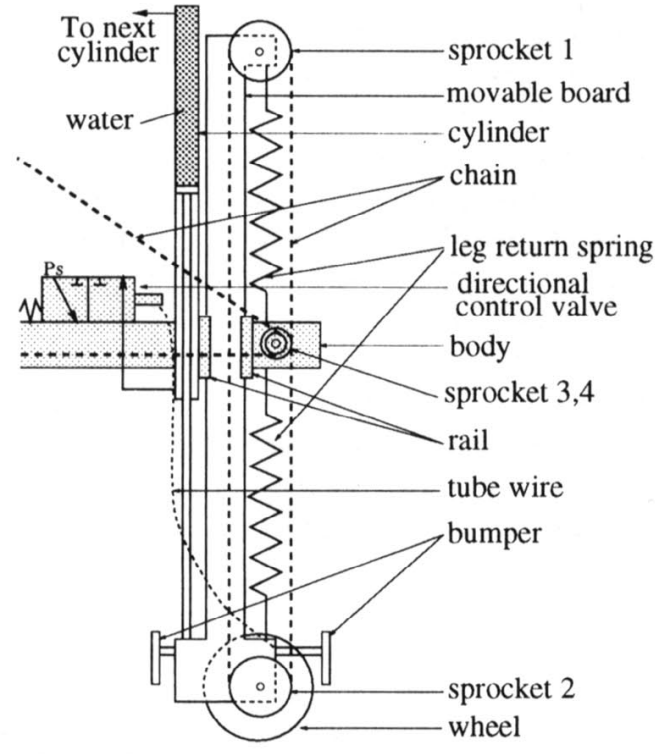

Figure 4 Structure of a leg

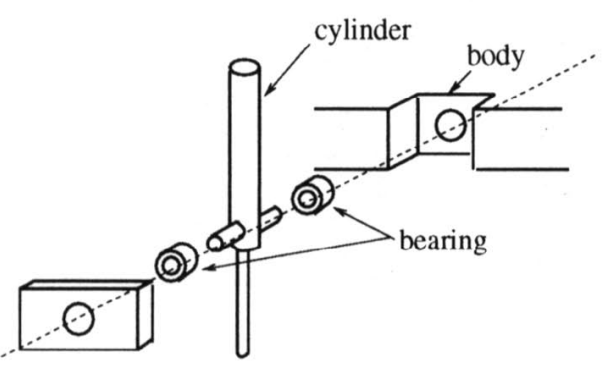

Figure 5 Installation method of a cylinder

\subsubsection{Structure of a bumper}

The structure of a bumper is shown in Figure 6 . Rollers are used to make the friction between a bumper and an obstacle small. A bumper is installed in front and back of a leg (front and back of a wheel).

At the usual time there is no high pressure water in the nod side of a cylinder while the head side is filled with water that acts as the medium of the communicating tubes. When a bumper is hit by an obstacle, the shape of the bumper will be changed from (a) to (b) as shown in Figure 6, and the tube wire can be pulled so as to open a directional control valve to let high pressure water flow into the rod side of the cylinder, which will lift the leg-wheel up to cross the obstacle. The bumper and the directional control valve can return to their original positions through return springs after having got over an obstacle, and the lifted wheel will also come down by both the weight of 
the robot and the leg return springs that are installed to the leg as shown in Figure 4.

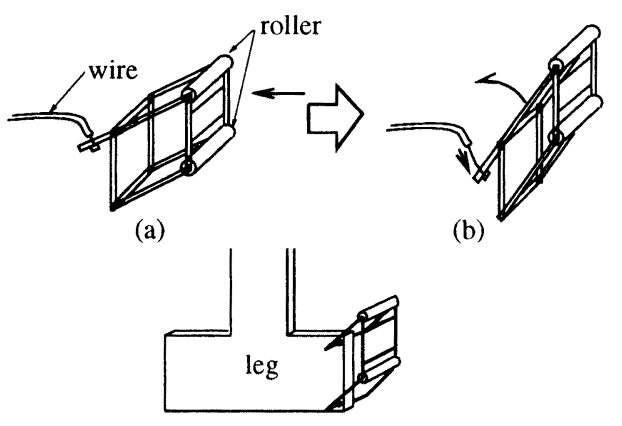

Figure 6 Structure of a bumper

\subsection{Parts arrangement of the robot}

The parts arrangement of the robot is shown in Figure 7. The robot is about $1200 \mathrm{~mm}$ in length, 700 $\mathrm{mm}$ in width and $1000 \mathrm{~mm}$ in height.

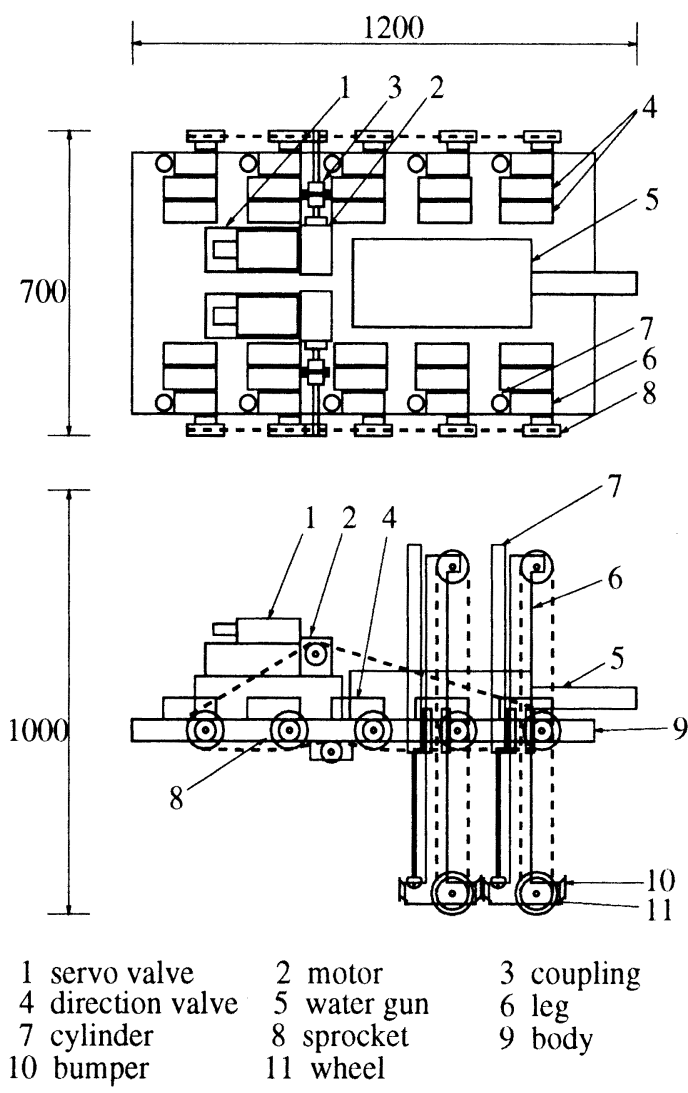

Figure 7 Parts arrangement of the robot

\section{Control system}

\subsection{Water hydraulic control system of the robot}

The water hydraulic control system can be divided into two parts by the dotted line as shown in Figure 8 . The lower part, which can be put on a place away from a fire spot, consists of a water hydraulic power unit and a computer-based controller. The upper part, which is a part of the robot. consists of water hydraulic servovalves, directional control valves. motors, etc.

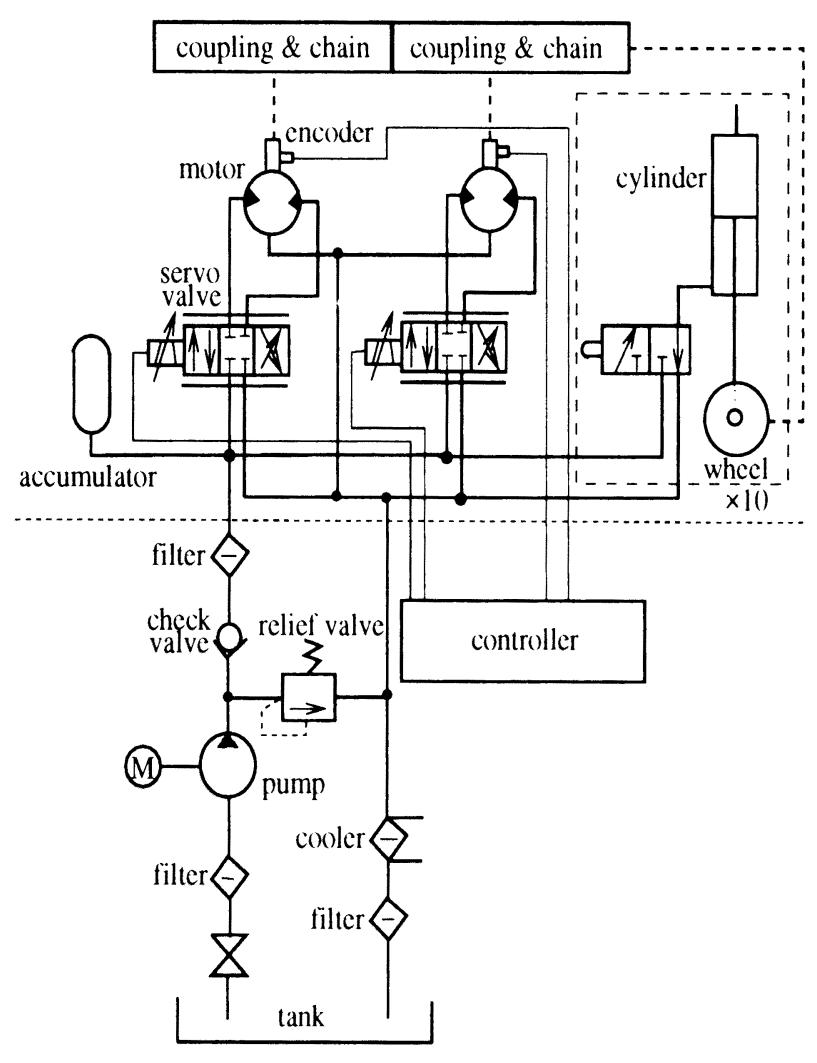

Figure 8 Water hydraulic control system

\subsection{Speed control of a motor}

\subsubsection{Experimental setup}

In order to find out whether a water hydraulic motor is usable for the robot or not. the experiment of speed control of Nessie water hydraulic motor MAH 6.3 was conducted. The experimental setup is shown in Figure 9. The rotational speed of the motor is controlled by a water hydraulic servovalve whose input comes from D/A 1. The motor output shaft is coupled with an electromagnetic particle brake whose torque is programmably controlled by the output voltage of 
D/A 2. At an end of the brake, a 2000 pulses/rev. encoder, which is used to detect the speed of the motor, is mounted.

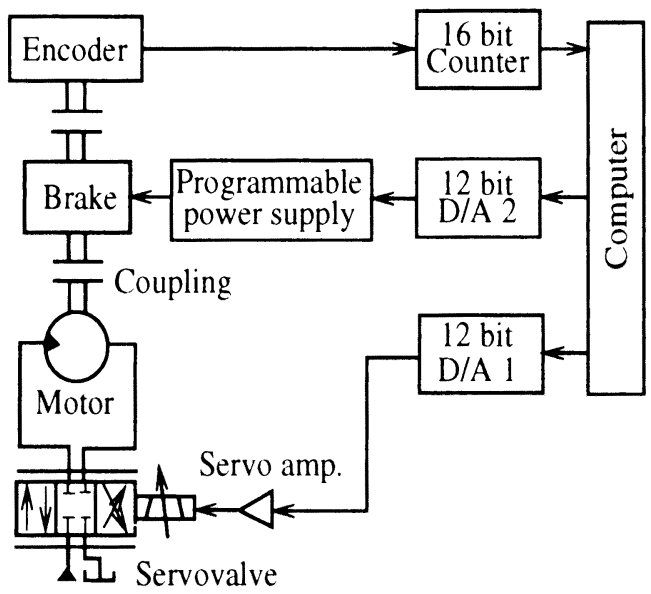

Figure 9 Schematic diagram of experimental setup

The block diagram of the motor speed control system is shown in Figure 10. PID control algorithm was used to control the rotational speed of the motor.

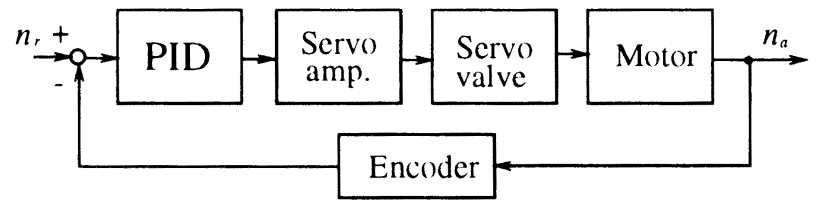

Figure 10 Block diagram of motor speed control system

\subsubsection{Speed control result}

One experimental result of speed control of the motor is shown in Figure 11. The supply pressure for the motor was set to $7 \mathrm{MPa}$. Here $n_{a}$ is the measured rotational speed of the motor, $n_{r}$ is the reference speed, and $T$ is the friction load that was applied to the motor. $T$ was chnaged by applying changeable output voltage to the electromagnetic particle brake through D/A 2. The value of $T$ was approximately calculated out according to the current-torque curve of the brake. The speed of the motor is controllable from $500 \mathrm{rpm}$ to 1500 rpm even though the load to the motor is changing, but the motor will not run when the speed is below $300 \mathrm{rpm}$, which therefore should be avoided in practical use.

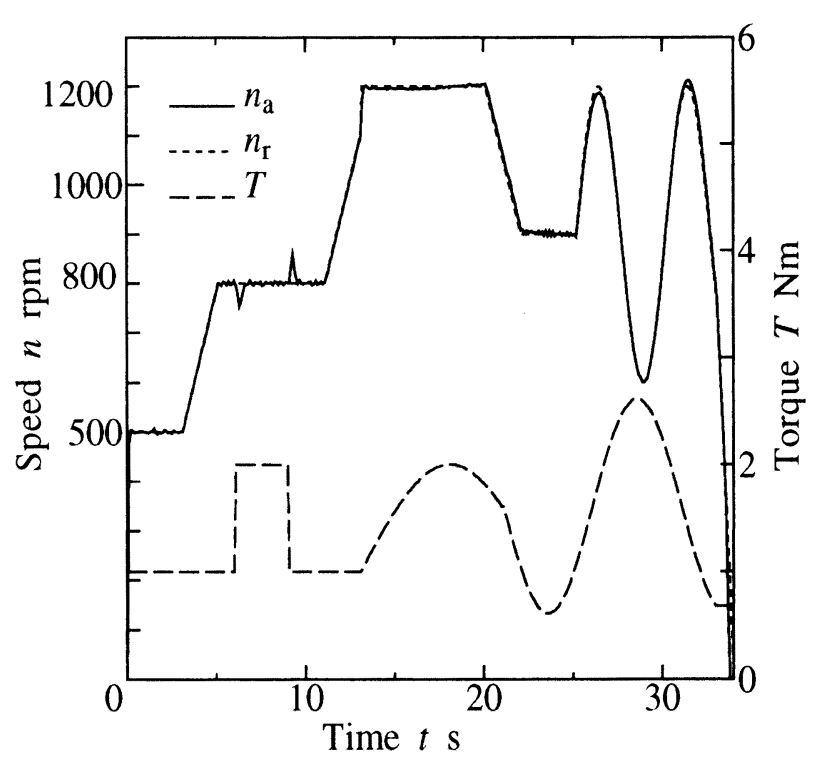

Figure 11 Result of motor speed control

\subsubsection{Maximum gear ratio between the motor and wheels}

The maximum torque of the motor can be up to 3.5 $\mathrm{Nm}$ while the supply pressure for the motor is $7 \mathrm{MPa}$. In order to decide the maximum gear ratio between the motor and wheels, the following worst situation for the robot to work at was considered.

The maximum weight of the robot that will be driven by one motor is $100 \mathrm{~kg}$, the robot is moving upward along a slope of 40 degrees, the friction factor between wheels and the ground is $0.25^{[4]}$, and the radius of a wheel is $0.075 \mathrm{~m}$.

The total torque necessary is $\left(100 \times 9.8 \times\left(\sin 40^{\circ}+\right.\right.$ $\left.\left.0.25 \times \cos 40^{\circ}\right)\right) \times 0.075=61.3 \mathrm{Nm}$. So the maximum gear ratio should be greater than $\frac{61.3}{3.5}=17.5$, and 20 was selected in practical use. The maximum running speed of the robot is about $2.1 \mathrm{~km} / \mathrm{h}$ when the gear ratio is 20 .

\subsubsection{Startup and stop pattern of motor speed}

The motor itself can be started up in about $0.2 \mathrm{~s}$ to a given speed ranging from $500 \mathrm{rpm}$ to $1500 \mathrm{rpm}$ and can be stopped within $0.2 \mathrm{~s}$ from $1500 \mathrm{rpm}$. But a sudden and great change of speed is not good for a chain, so the startup and stop of motor should be carried out gradually. One possible speed pattern is shown in Figure 12. Both the startup time and the stop time are set to $1 \mathrm{~s}$ respectively. Here again PID control algorithm was used to control the rotational speed of the motor. Because the motor can not run 
below $300 \mathrm{rpm}$, the lowest reference speed was set to $300 \mathrm{rpm}$ for startup. The motor can be completely stopped within 12 rotations. That is to say, the robot will move about $0.28 \mathrm{~m}$ at most before it stops when the gear ratio of 20 and the wheel radius of $0.075 \mathrm{~m}$ are considered. Actually, the running speed of the robot is not always the highest, so the robot will be stopped in shorter than $0.28 \mathrm{~m}$. Some other speed patterns for stopping the robot are also possible.

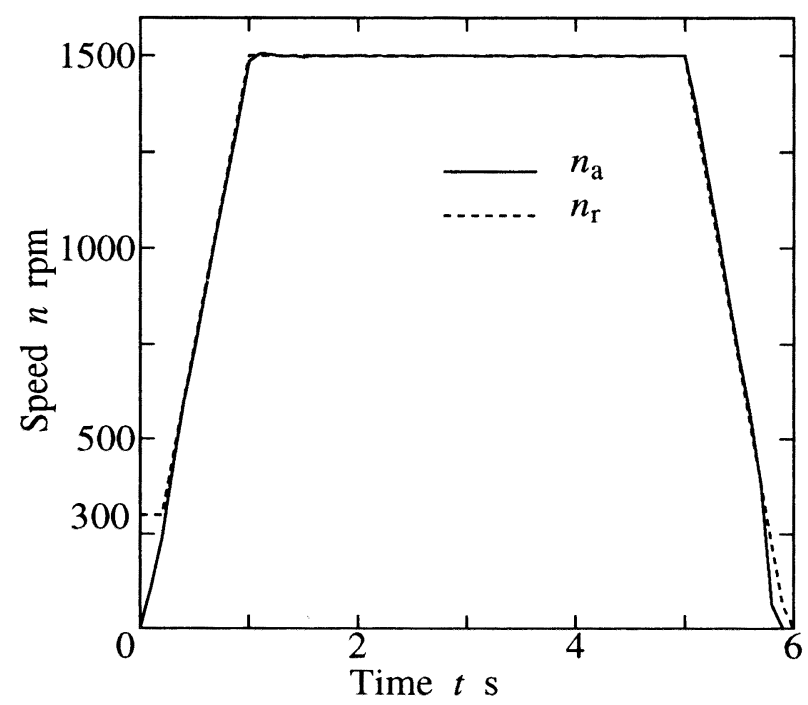

Figure 12 Startup and stop pattern of motor speed

\section{Conclusion}

In this paper, a water hydraulic fire-fighting robot was proposed. The proposed robot, which mainly consists of 10 leg-wheels, can run along flat ground, climb steps, go directly into firing places. The wheels can run fast along flat places and the legs can go up and down. The wheels are driven by two water hydraulic motors and the legs are driven by water hydraulic cylinders. The mechanism of the robot, such as the drive system, the leg structure, the parts arrangement, etc., was discussed. The speed control experiment was done by use of Nessie water hydraulic motor MAH 6.3. The experimental result is that the speed of the motor is controllable from $500 \mathrm{rpm}$ to $1500 \mathrm{rpm}$ even though the load to the motor is changing.

The whole robot is being built.

\section{REFERENCES}

1. K. Yamamoto, Robots Working in Extremely Dangerous Situations, Industry Investigating Committee, 1992, pp.327
2. E. Trostmann, Water Hydraulics Control Technology, Marcel Dekker, Inc., 1996

3. Y. Kumakiri, Mobile Robots, TOPPAN. 1993

4. M. Ito, Easily understood Mechanism Design. Industry Investigating Committee, 1988, pp.28 